\title{
Hysteresis in a bimetallic holmium complex : A synergy between electronic and nuclear magnetic interactions
}

\section{Viciano-Chumillas, Marta}

2017-12-21

Viciano-Chumillas , M , Koprowiak , F, Mutikainen , I, Wernsdorfer , W , Mallah , T \& Bolvin , H 2017 , ' Hysteresis in a bimetallic holmium complex : A synergy between electronic and nuclear magnetic interactions ' , Physical Review B , vol. 96 , no. 21 , 214427 . https://doi.org/10.1103/PhysRevB.96

http://hdl.handle.net/10138/231026

https://doi.org/10.1103/PhysRevB.96.214427

cc_by

publishedVersion

Downloaded from Helda, University of Helsinki institutional repository.

This is an electronic reprint of the original article.

This reprint may differ from the original in pagination and typographic detail.

Please cite the original version. 


\title{
Hysteresis in a bimetallic holmium complex: A synergy between electronic and nuclear magnetic interactions
}

\author{
Marta Viciano-Chumillas \\ Institut de Chimie Moléculaire et des Matériaux d'Orsay, CNRS, Université de Paris-Sud 11, 91405 Orsay Cedex, France
}

Florian Koprowiak

Laboratoire de Chimie et Physique Quantiques, IRSAMC Université Toulouse 3, 118 route de Narbonne, 31062 Toulouse, France

Ilpo Mutikainen

Laboratory of Inorganic Chemistry, Department of Chemistry (A. I. Virtasen aukio 1), P.O. Box 55, FI-00014, University of Helsinki, Finland

Wolfgang Wernsdorfer

Institut Néel, CNRS, Université Joseph Fournier, Grenoble Cedex 9, France

and Institute of Nanotechnology, Karlsruhe Institute of Technology, Germany

Talal Mallah*

Institut de Chimie Moléculaire et des Matériaux d'Orsay, CNRS, Université de Paris-Sud 11, 91405 Orsay Cedex, France

Hélène Bolvin ${ }^{\dagger}$

Laboratoire de Chimie et Physique Quantiques IRSAMC Université Toulouse 3, 118 route de Narbonne, 31062 Toulouse, France

(Received 21 July 2017; revised manuscript received 20 October 2017; published 21 December 2017)

\begin{abstract}
We report a bimetallic holmium(III) complex showing a S-shaped magnetic hysteresis at low temperature. The complex is investigated by x-ray crystallography, magnetometry, single crystal microsquid measurements, and first-principles calculations. A model Hamiltonian including electronic and nuclear magnetic moments is used to fit all experimental data. We conclude that the Ho(III) may be described as non-Kramers doublets with respective gaps of $\Delta_{A}=0.8$ and $\Delta_{B}=10 \mathrm{~cm}^{-1}$ and that there is a small ferromagnetic coupling of $J=1 \mathrm{~cm}^{-1}$ $\left(\hat{\mathcal{H}}_{S}=-J \boldsymbol{S}_{A} \cdot \boldsymbol{S}_{B}\right)$. As in previous works, the hysteresis arise from the hyperfine structure of the Ho(III) ions. The S-shaped form of the hysteresis reflects the avoided crossing of the electronic states in the non-Kramers doublets.
\end{abstract}

DOI: 10.1103/PhysRevB.96.214427

\section{INTRODUCTION}

The lanthanide based complexes present a large anisotropic magnetic moment in their ground state due their unquenched orbital moment which often leads to a single-molecule magnet (SMM) behavior [1]. This class of molecules is promising for molecular spintronics and opens the doors to quantum computing [2-4]. The two-level system (arising from the two directions of the magnetic moment) of each molecule may act as a basic unit, namely as a qubit. In order to access qugates, one should design molecules with two interacting qubits [5-8] that can be lanthanide ions. But due to the innershell character of the magnetic $4 f$ electrons, the interaction between neighboring magnetic centers is usually very weak [9], and designing binuclear lanthanide-containing complexes with sizable exchange coupling is a challenge. SMM behavior mostly take place in Kramers ions such as dysprosium complexes but some cases are known for the non-Kramers ions of holmium complexes. Ishikawa et al. [10] have observed the stairlike magnetization hysteresis loop in a phtalocyano

\footnotetext{
*talal.mallah@u-psud.fr

†bolvin@irsamc.ups-tlse.fr
}

Ho(III) complex arising from the hyperfine structure of the Ho(III) ion. The same type of hysteresis has been recently evidenced by Chen et al. in a pentagonal Ho(III) complex [11]. The study of the interplay of the SMM behavior in dinuclear complexes is quite recent and is mainly focused on Dy-containing complexes [12-19]. Magnetization hysteresis loops have been observed in a series of $\operatorname{Ln}$ (III) [20] and in several bimetallic Dy(III) [21-24]. However, to the best of our knowledge, no magnetic hysteresis loop was reported for binuclear Ho(III)-containing complexes [25]. We show that despite the nonaxial crystal field of the two Ho(III) ions that should lead to a large mixing between their two low-lying $M_{J}$ levels and thus to the absence of a magnetic hysteresis loop, the local hyperfine coupling together with the exchange coupling are responsible of the blocking of the magnetization in the binuclear complex.

In this article we present a binuclear Ho(III) complex of formula $\left[\mathrm{Ho}_{2} \mathrm{~L}_{3}\left(\mathrm{CH}_{3} \mathrm{OH}\right)\right]$ where $\mathrm{H}_{2} \mathrm{~L}$ is depicted in Fig. 1 . We detail its synthesis, its characterization by $\mathrm{x}$-ray crystallography, magnetochemistry, single crystal microsquid, and the first-principles method SO-CASSCF. A model Hamiltonian is proposed and its parameters are either deduced from SO-CASSCF calculations or fitted to reproduce the magnetic data. The complex shows a hysteresis loop at low temperature. 


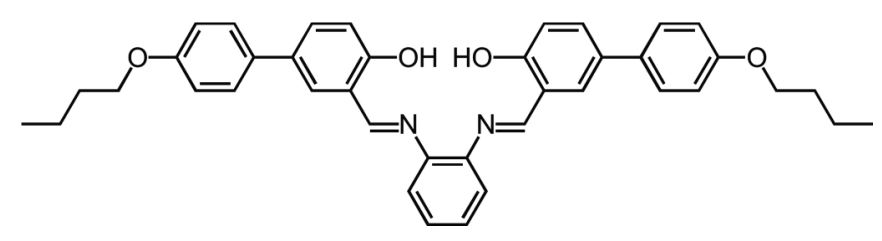

FIG. 1. $\mathrm{H}_{2} \mathrm{~L}$ ligand.

With the help of quantum chemical tools and the model Hamiltonian, we analyze the effect of the interplay between the electronic magnetic moments and the hyperfine structure; we conclude that the hysteresis is driven by the hyperfine sublevels of the two Ho(III) ions and the electronic splitting of the non-Kramers doublets and the magnetic coupling between the two magnetic centers determine the shape of the loop. Finally, this model Hamiltonian allows unraveling the role of the electronic and nuclear degrees of freedom.

\section{EXPERIMENTAL AND COMPUTATIONAL RESULTS}

\section{A. Crystal structure and magnetometry}

The x-ray crystal structure shows that the two Ho(III) ions are separated by $3.841 \AA$ within the triple-decker-like binuclear complex (see Fig. 2 and the Supplemental Material [26]). The two Ho(III) sites are not equivalent; Hol is octacoordinated while $\mathrm{Ho} 2$ is heptacoordinated. They are bridged by two oxygen atoms belonging to the central $\mathrm{L}^{2-}$ ligand. The static magnetic behavior of the complex was investigated. The room temperature $\chi_{M} T$ value of $26.7 \mathrm{~cm}^{3} \mathrm{~K} \mathrm{~mol}^{-1}$ (where $\chi_{M}$ is the molar magnetic susceptibility and $T$ is the temperature) is in good agreement with the expected value for two noninteracting Ho(III) ions (term ${ }^{5} I_{8}, g_{J}=5 / 4 \chi_{M} T=28.1 \mathrm{~cm}^{3} \mathrm{~K} \mathrm{~mol}^{-1}$ ) and the decrease at low temperature is due to a gradual depopulation of the Starks levels (see Fig. 3). Molecular magnetization $M$ as a function of applied magnetic field shows a smooth increase and the saturation is not reached at $6 \mathrm{~T}$ (see inset of Fig. 3).

The magnetic behavior was investigated on a single crystal using a microsquid set up that allows us to cool down to $30 \mathrm{mK}$ [27]. The magnetic hysteresis appears below $0.15 \mathrm{~K}$. The hysteresis loop was then measured at $T=0.03 \mathrm{~K}$ with different sweep rates of the magnetic field ranging from 0.28 to $0.001 \mathrm{~T} / \mathrm{s}$ (see Fig. 4). Upon decreasing the sweep rate from 0.280 to $0.001 \mathrm{~T} / \mathrm{s}$, the coercive field decreases from 770 to $257 \mathrm{Oe}$, which is the signature of a quantum tunneling of the magnetization (QTM) and not of a collective magnetic order.

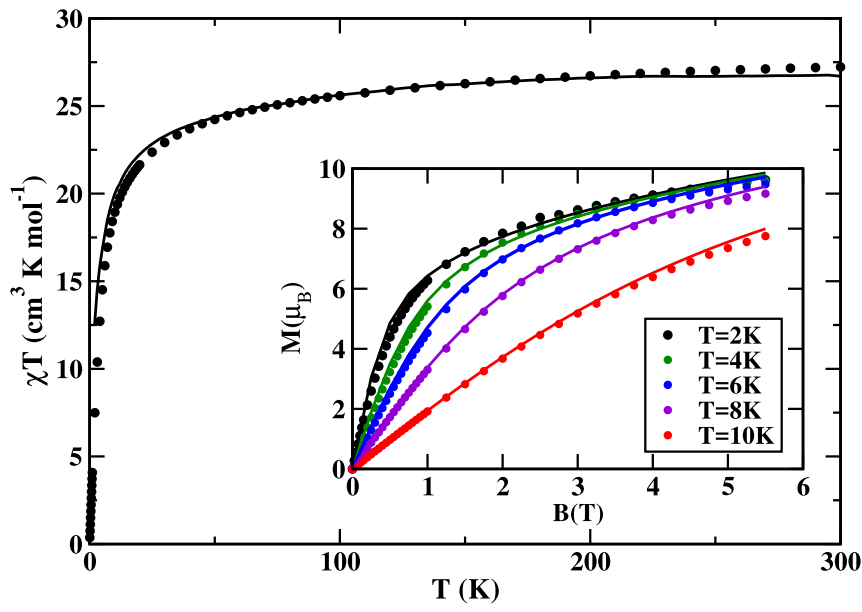

FIG. 3. $\chi_{M} T$ versus $T$. The inset shows the magnetization as a function of magnetic field for different temperatures; points: experiment; solid line: combined SO-CASSCF and fit.

\section{B. First-principles calculations}

SO-CASSCF calculations [28-33] were performed on Ho1 Lu and LuHo2 species, noted complexes $\mathbf{A}$ and $\mathbf{B}$, respectively, where one $\mathrm{Ho}$ (III) ion is replaced by a diamagnetic $\mathrm{Lu}(\mathrm{III})$ ion. According to SO-CASSCF calculations (see the Supplemental Material for more information [26]), the ground term of the free ion ${ }^{5} I_{8}$ splits into 17 nondegenerate states by 340 and $400 \mathrm{~cm}^{-1}$ for $\mathbf{A}$ and $\mathbf{B}$, respectively, and in both species, the first excited state lies at $11 \mathrm{~cm}^{-1}$ above the ground state. Since none of the states are degenerate, the magnetization arises through second order Zeeman interaction between the Starks levels. At low temperature, magnetic properties are mostly determined by the two lowest states, which may be described as a non-Kramers doublet. The $\mathbf{g}$ matrix is calculated by the same method as for Kramers doublets [34] but in the case of a non-Kramers doublet, only one of the three $g$ factors is nonzero; within this model space restricted to two states, a magnetization is induced by the magnetic field when applied along the $Z$ direction and there is no magnetization in the two other directions. The SO-CASSCF calculations give $g_{A}=$ 15.0 and $g_{B}=15.4$ for $\mathbf{A}$ and $\mathbf{B}$, with, respectively, an angle of $57^{\circ}$ and $20^{\circ}$ with the metal-metal axis. The two local magnetic moments $\boldsymbol{\mu}_{A}$ and $\boldsymbol{\mu}_{B}$ form an angle of $37^{\circ}$ (see Fig. 5). The two ground states have similar decomposition in terms of $M_{J}$ [35], with a strong mixing of $M_{J}= \pm 8, \pm 7, \pm 5$. The $M$ vs $B$ and $\chi T$ vs $T$ curves calculated with SO-CASSCF [36] do not fit well with the experimental data, and it was necessary to

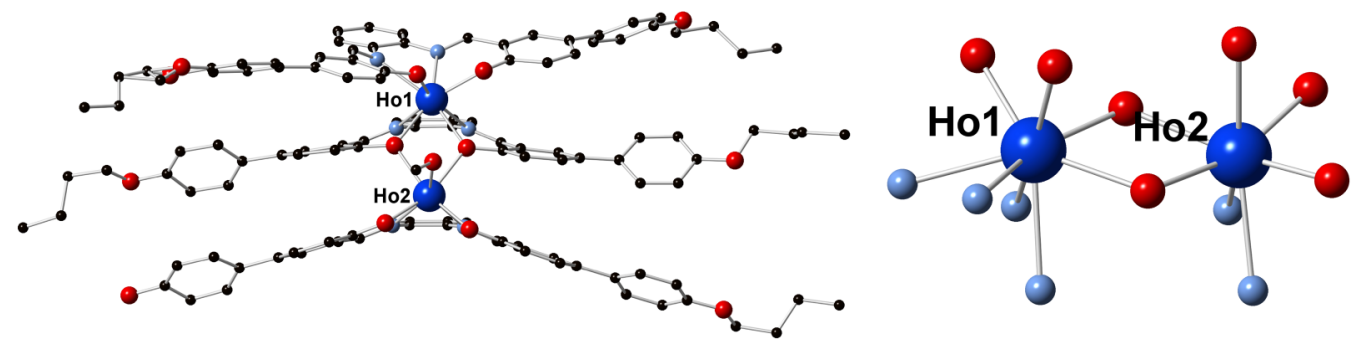

FIG. 2. View of the structure of the binuclear complex $\left[\mathrm{Ho}_{2} \mathrm{~L}_{3}(\mathrm{MeOH})\right]$ complex (left) and of the coordination sphere of the Ho(III) ions (right); $\mathrm{H}$ atoms were removed for clarity. Color code: Ho (dark blue), $\mathrm{N}$ (light blue), O (red), and C (black). 

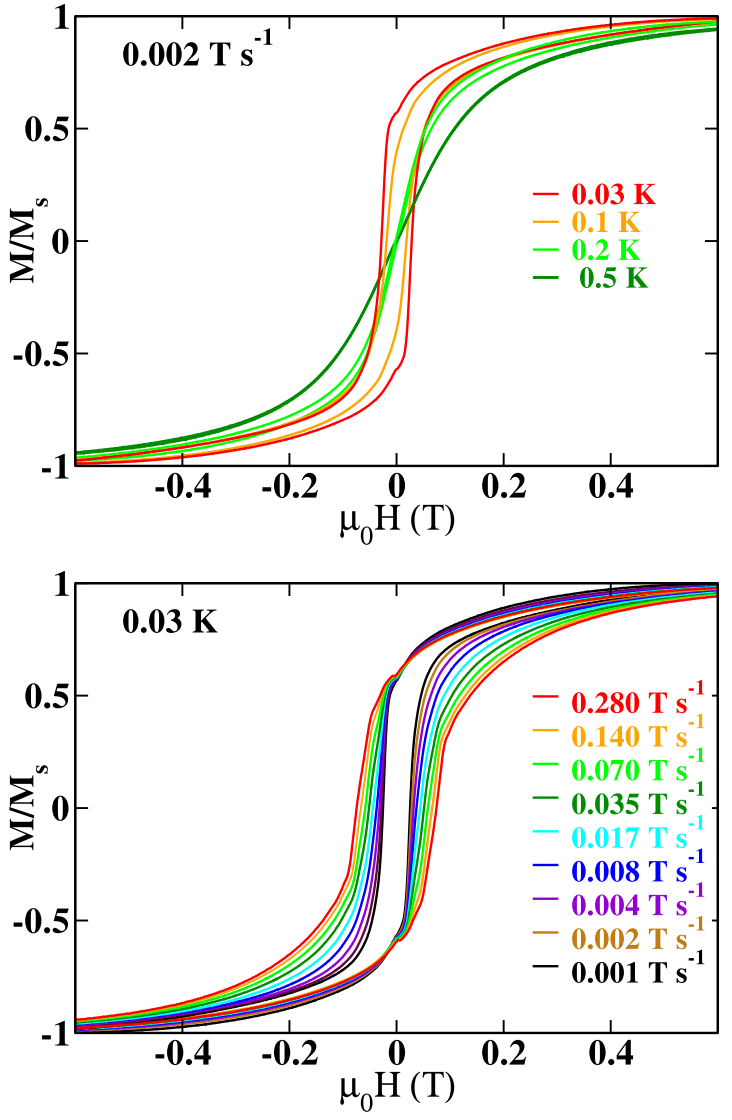

FIG. 4. Magnetization loops at different temperatures with a sweep rate of $0.002 \mathrm{~T} / \mathrm{s}$ (up) and at $T=0.03 \mathrm{~K}$ at different sweep rates of the magnetic field (down).

adjust the two energy gaps $\Delta_{A}$ and $\Delta_{B}$ to split the other excited states and to add a magnetic coupling in order to reproduce the experimental data. The best adjustment is provided with $\Delta_{A}=0.8 \mathrm{~cm}^{-1}, \Delta_{B}=10 \mathrm{~cm}^{-1}$, and $J=1 \mathrm{~cm}^{-1}$ (see Fig. 3). These gaps are larger than those previously determined in

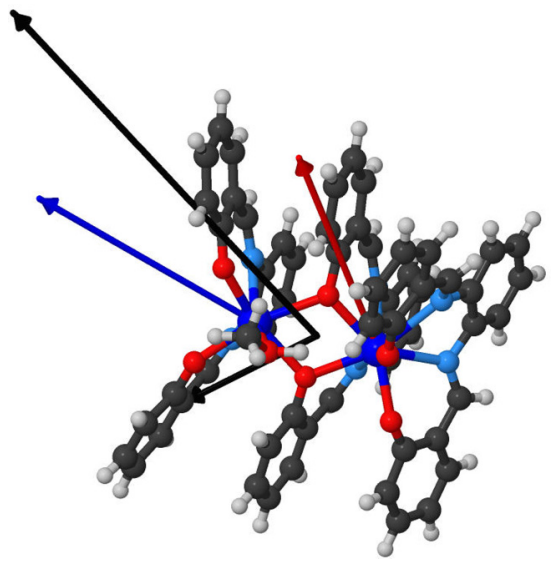

FIG. 5. The $\left[\mathrm{Ho}_{2} \mathrm{~L}_{3}\left(\mathrm{CH}_{3} \mathrm{OH}\right)\right]$ complex. Local magnetic moments calculated with SO-CASSCF for sites A (red) and B (blue) corresponding to Ho1 Lu and $\mathrm{LuHo} 2$, respectively, and for Ho1Ho2 (black). The arrows are in the direction of the moment and the length is proportional to $g_{I}$.
Ho(III) complexes which are less than $0.3 \mathrm{~cm}^{-1}[10,11,37,38]$. In the previous studies, the environment around the Ho(III) ion was symmetrical and the ground state was pure in terms of $M_{J}$. Even if the SO-CASSCF calculations do not match perfectly the experimental curves, they serve as a starting point for the characterization of the two magnetic centers: the nature of the ground doublet and its magnetization (both modulus and direction) are supposed to be correctly determined, and we adjusted as few parameters as possible. They permit an estimation of the magnetic characteristics of each Ho(III) site. According to the local magnetic moments determined by the SO-CASSCF calculation, the magnetic dipolar interaction between the two centers is slightly ferromagnetic $\left(\approx 0.3 \mathrm{~cm}^{-1}\right)$ because of the orientation of the two local moments and the fit of the magnetic data leads to an additional coupling of $J=1 \mathrm{~cm}^{-1}$ [9] (see the Supplemental Material [26]). Since one of the energy gaps needed to be strongly readjusted, this partition between dipolar and exchange interactions should be taken with care and one should better remember that the total interaction is slightly ferromagnetic. Anyhow, it will be shown in the following that this coupling has only a small impact on the shape of the hysteresis curve.

\section{SPIN HAMILTONIAN}

\section{A. Electronic spin Hamiltonian}

The spin Hamiltonian for a non-Kramers doublet takes the form for site $\mathbf{A}$, with the zero-field part $\hat{\mathcal{H}}_{A}^{0}$ and the Zeeman term $\hat{\mathcal{H}}_{A}^{Z e}[39,40]$ :

$$
\hat{\mathcal{H}}_{A}=\hat{\mathcal{H}}_{A}^{0}+\hat{\mathcal{H}}_{A}^{Z e}=\Delta_{A} \mathbb{S}_{A}^{Z_{A}}+\mu_{B} g_{A} \mathbb{S}_{A}^{X_{A}} B^{Z_{A}},
$$

where $\Delta_{A}$ and $g_{A}$ are the energetic splitting and the $g$ factor of the doublet and $\mathbb{S}$ is a pseudospin operator with components $\mathbb{S}^{X}, \mathbb{S}^{Y}, \mathbb{S}^{Z}$, and $\mathbb{S}=1 / 2 . \mu_{B}$ is the Bohr magneton and $B^{Z_{A}}$ is the external magnetic field along the $Z_{A}$ direction. The eigenvectors of $\hat{\mathcal{H}}_{A}^{0}$ are the two zero-field states $|1\rangle_{A}$ and $|2\rangle_{A}$ with vanishing magnetic moments and a energy gap of $\Delta_{A}$. The magnetization arises from the coupling between these two states such that the Zeeman term $\hat{\mathcal{H}}_{A}^{Z e}$ has only off-diagonal elements in the basis of zero-field states and is described by $\mathbb{S}_{A}^{X_{A}}$. The local magnetic states are $| \pm\rangle_{A}=1 / \sqrt{2}\left(|1\rangle_{A} \pm|2\rangle_{A}\right)$ with respective magnetic moments $\pm \frac{1}{2} \mu_{B} g_{A}$. A similar Hamiltonian is obtained for site $\mathbf{B}$.

The coupling Hamiltonian has two contributions (i) the magnetic dipolar one

$$
\hat{\mathcal{H}}^{\mathrm{dip}}=\frac{\mu_{0}}{4 \pi R^{3}}\left(\boldsymbol{\mu}_{A} \cdot \boldsymbol{\mu}_{B}-3 \mu_{A}^{0} \mu_{B}^{0}\right),
$$

where $R$ is the intermetallic distance, 0 is the intermetallic direction, and $\mu_{0}$ is the magnetic constant. Due to the axial nature of the moments, the Hamiltonian of Eq. (2) may be written as an Ising Hamiltonian with the two local pseudospins

$$
\hat{\mathcal{H}}^{\mathrm{dip}}=-J \mathbb{S}_{A}^{Z_{A}} \mathbb{S}_{B}^{Z_{B}},
$$

and according to the SO-CASSCF calculations on the monomers, $J^{\text {dip }}=0.3 \mathrm{~cm}^{-1}$. The exchange interaction is carried by the spin densities and is described by a HeisenbergDirac-Van Vleck (HDVV) Hamiltonian

$$
\hat{\mathcal{H}}^{\text {heis }}=-J^{\text {heis }} \boldsymbol{S}_{A} \cdot \boldsymbol{S}_{B}
$$




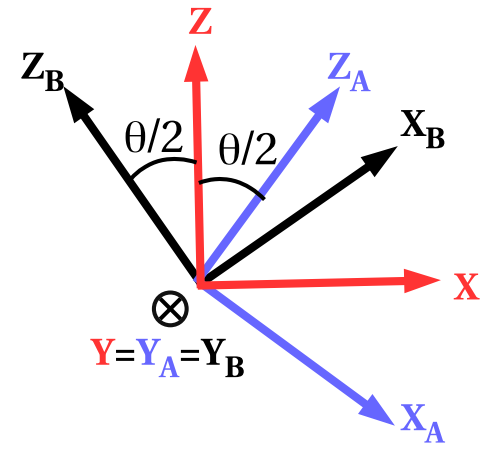

FIG. 6. Representation of the three Cartesian coordinates frames: $Z_{A(B)}$ is the direction of $\mu_{A(B)}, Z$ is the bisector of $Z_{A}, Z_{B}$, and $Y$ is common to the three frames.

involving the local spin operators $\boldsymbol{S}_{A(B)}$; due to the anisotropic local magnetic moments, it may be expressed by an Ising Hamiltonian as well. Both interaction terms, in the space of the ground doublet, may be grouped as

$$
\hat{\mathcal{H}}_{A B}^{0}=-J \mathbb{S}_{A}^{Z_{A}} \mathbb{S}_{B}^{Z_{B}} .
$$

Finally, the total zero-field Hamiltonian

$$
\hat{\mathcal{H}}_{\mathrm{el}}=\hat{\mathcal{H}}_{A}+\hat{\mathcal{H}}_{B}+\hat{\mathcal{H}}_{A B}^{0}
$$

has the following matrix representation in the basis $| \pm \pm\rangle=$ $| \pm\rangle_{A} \otimes| \pm\rangle_{B}$ and $| \pm \mp\rangle=| \pm\rangle_{A} \otimes|\mp\rangle_{B}$ :

\begin{tabular}{cccc}
$|++\rangle$ & $|+-\rangle$ & $|-+\rangle$ & $|--\rangle$ \\
\hline$-J / 4$ & $\Delta_{B} / 2$ & $\Delta_{A} / 2$ & 0 \\
$\Delta_{B} / 2$ & $J / 4$ & 0 & $\Delta_{A} / 2$ \\
$\Delta_{A} / 2$ & 0 & $J / 4$ & $\Delta_{B} / 2$ \\
0 & $\Delta_{A} / 2$ & $\Delta_{B} / 2$ & $-J / 4$
\end{tabular}.

Since the Hamiltonians of the two complexes $\mathbf{A}$ and $\mathbf{B}$ are not expressed in the same system of coordinates, it is convenient to introduce a system of coordinates for the dinuclear complex. $Z_{A(B)}$ is the direction of the magnetic moment of the doublet of $\mathbf{A}(\mathbf{B}) . X$ and $Z$ are the bisectors of directions $Z_{A}$ and $Z_{B}, Z$ being the bisector of the acute angle and $X$ for the obtuse one (see Fig. 6). The states of the dinuclear species are built as $| \pm \pm\rangle=| \pm\rangle_{A} \otimes| \pm\rangle_{B}$, where $| \pm\rangle_{A(B)}$ are the two eigenstates of $\hat{\mathcal{H}}_{A(B)}^{Z e}, \hat{\mathcal{H}}_{A(B)}^{Z e}| \pm\rangle_{A(B)}=$

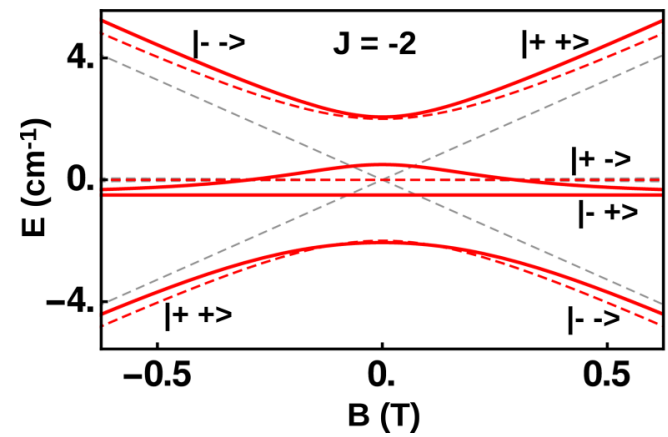

$\pm 1 / 2 \mu_{B} g_{A(B)} B^{Z}$, namely the states with local magnetization up and down.

The total magnetic moment $\boldsymbol{\mu}$ of the dinuclear complex is the sum of the two local magnetic moments $\mu_{A}$ along $Z_{A}$ and $\boldsymbol{\mu}_{B}$ along $Z_{B}$. Its component along the $\mathrm{Z}$ axis is

$$
\mu^{Z}=\mu_{A}^{Z}+\mu_{B}^{Z}=-\frac{1}{2} \mu_{B}\left(\cos \frac{\theta}{2} g_{A} \mathbb{S}_{A}^{Z_{A}}+\cos \frac{\theta}{2} g_{B} \mathbb{S}_{B}^{Z_{B}}\right),
$$

where $\theta / 2$ is the angle between $Z$ and both $Z_{A}$ and $Z_{B}$. The component along $X$ is

$$
\mu^{X}=\mu_{A}^{X}+\mu_{B}^{X}=-\frac{1}{2} \mu_{B}\left(\sin \frac{\theta}{2} g_{A} \mathbb{S}_{A}^{Z_{A}}-\sin \frac{\theta}{2} g_{B} \mathbb{S}_{B}^{Z_{B}}\right),
$$

and the component along $Y$ vanishes. The eigenvalues and eigenstates for these operators are

$$
\begin{aligned}
M^{Z} & =\left\langle++\left|\hat{\mu}^{Z}\right|++\right\rangle=-\left\langle--\left|\hat{\mu}^{Z}\right|--\right\rangle \\
& =\frac{1}{2} \mu_{B}\left(g_{A}+g_{B}\right) \cos \theta / 2, \\
m^{Z} & =\left\langle+-\left|\hat{\mu}^{Z}\right|+-\right\rangle=-\left\langle-+\left|\hat{\mu}^{Z}\right|-+\right\rangle \\
& =\frac{1}{2} \mu_{B}\left(g_{A}-g_{B}\right) \cos \theta / 2, \\
m^{X} & =\left\langle++\left|\hat{\mu}^{X}\right|++\right\rangle=-\left\langle--\left|\hat{\mu}^{X}\right|--\right\rangle \\
& =\frac{1}{2} \mu_{B}\left(g_{A}-g_{B}\right) \sin \theta / 2, \\
M^{X} & =\left\langle+-\left|\hat{\mu}^{X}\right|+-\right\rangle=-\left\langle-+\left|\hat{\mu}^{X}\right|-+\right\rangle \\
& =\frac{1}{2} \mu_{B}\left(g_{A}+g_{B}\right) \sin \theta / 2 .
\end{aligned}
$$

The Zeeman diagram with a field along $Z$ is shown in Fig. 7 in the case of symmetrical Ho(III) sites and for both ferromagnetic and antiferromagnetic couplings. These curves show that the magnetic behavior of the dinuclear complex may be described as the avoided crossing between the $|++\rangle$ and the $|--\rangle$ states due the ZFS of the local doublets $\Delta_{A}$ and $\Delta_{B}$. The two $|+-\rangle$ and $|-+\rangle$ states are entangled and are mostly nonmagnetic due to the compensation of the local magnetic moments in the up-down conformation. The easy axis of magnetization for the dinuclear complex is the bisector of the two local magnetic moments. The magnetic coupling $J$ affects mostly the two $|+-\rangle$ and $|-+\rangle$ states with a small

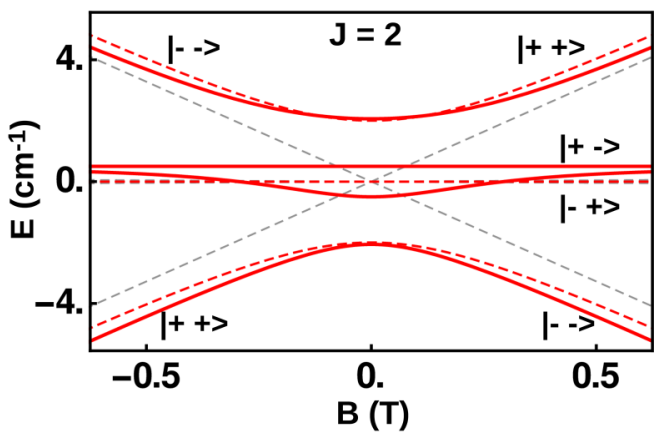

FIG. 7. Zeeman diagram with a field in direction $Z$ for the dinuclear complex. In solid red, $\Delta_{A}=\Delta_{B}=2 \mathrm{~cm}^{-1}, M^{Z}=15, m^{Z}=0$, and $J=-2 \mathrm{~cm}^{-1}$ (left) or $J=2 \mathrm{~cm}^{-1}$ (right); in dashed red, $J=0$ and in dashed gray, $\Delta_{A}=\Delta_{B}=0 \mathrm{~cm}^{-1}$ and $J=0$. 

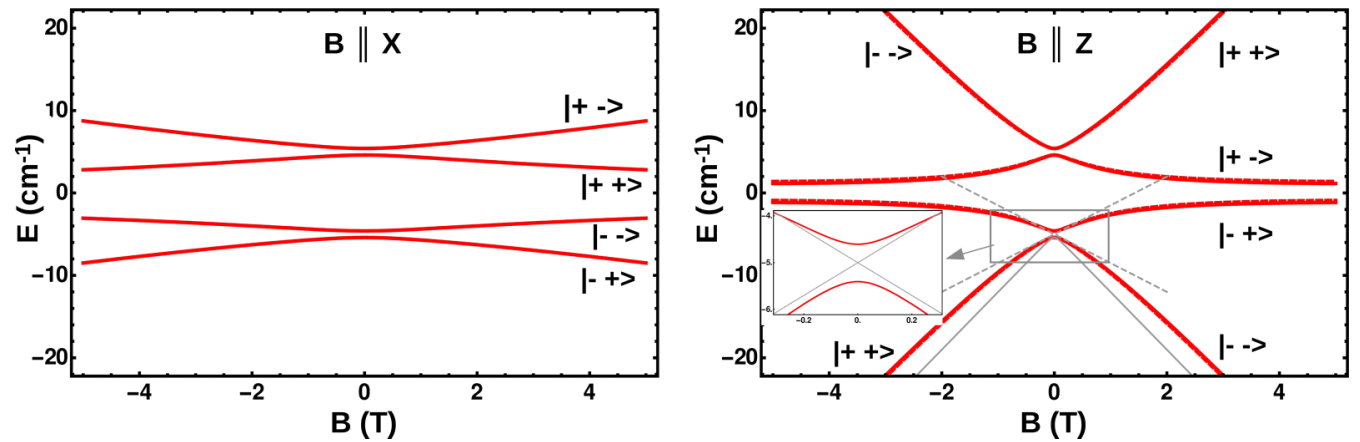

FIG. 8. Electronic Zeeman levels for the dinuclear complex for a magnetic field in directions $X$ (left) and $Z$ (right). In solid red, $\Delta_{A}=0.8 \mathrm{~cm}^{-1}, \Delta_{B}=10 \mathrm{~cm}^{-1}, g_{A}=15.0, g_{B}=15.4, \theta=37^{\circ}$, and $J=1 \mathrm{~cm}^{-1}$; in gray, the mononuclear complex with $\Delta=0$ and $g=M^{Z}$ (dashed) and $\Delta=0.8 \mathrm{~cm}^{-1}$ and $g=2 M^{Z}$ (solid).

gap opening between the two curves at small field. Its effect on the lower curve is to decrease the magnetization when $J<0$ and inversely, to increase it when $J>0$.

With the parameters deduced from the ab initio calculations on the complexes $\mathbf{A}$ and $\mathbf{B}, g_{A}=15, g_{B}=15.4$, and $\theta=37^{\circ}$, one gets $m^{X}=0.03, M^{X}=2.4, m^{Z}=0.2$, and $M^{Z}=15 \mu_{B}$. The magnetization is mostly borne by the $|++\rangle$ and $|--\rangle$ states along the $Z$ axis because $\theta$ is relatively small and $g_{A} \approx g_{B}$. The Zeeman diagrams obtained by diagonalizing the Hamiltonian of Eq. (6) in the $| \pm \pm\rangle,| \pm \mp\rangle$ basis set are shown in Fig. 8. Since $\Delta_{B}=10 \mathrm{~cm}^{-1}$ is much larger than $\Delta_{A}=$ $0.8 \mathrm{~cm}^{-1}$, the four states are split into two pseudodoublets separated by $\Delta_{B}$ and in each doublet there is an avoided crossing due to $\Delta_{A}$. At very low field, the magnetization of the ground doublet is about $M^{Z} / 2$ and it increases to $M^{Z}$ at higher fields.

\section{B. Spin Hamiltonian with hyperfine coupling}

It has been shown in previous studies that the hyperfine coupling (HFC) of the Ho(III) ion plays the key role in the hysteresis in holmium complexes [10,11]. Since the hysteresis appears in the sub-Kelvin region, the electronic Hamiltonian of Eq. (6) in the space of the two first doublets is sufficient and the other states will be omitted in the following.

For the free ion, in the LS coupling scheme, the interaction between the nuclear magnetic moment and the magnetic field of the electrons may be written as [41]

$$
\hat{\mathcal{H}}^{\mathrm{HFC}}=A_{J} \boldsymbol{J} \cdot \boldsymbol{I},
$$

where $\boldsymbol{I}$ is the spin operator of the nucleus and $\boldsymbol{J}$ is the total electronic angular momentum. The natural abundance for holmium is $100 \%{ }^{165} \mathrm{Ho}$ with $I=7 / 2$. The Hamiltonian of Eq. (14) applies in the $2 J+1$ manifold of the ${ }^{5} I_{8}$ term. Its projection in the subspace of the ground doublet may be expressed using the pseudospin operator $\hat{\mathcal{H}}^{\mathrm{HFC}}=\mathbb{S} \cdot \mathbf{A} \cdot \mathbb{S}$, where $\mathbf{A}$ is the hyperfine coupling tensor, the tensors $\mathbf{g}$ and A have the same principal axes. Consequently, in the present case, $\mathbf{A}$ is axial as $\mathbf{g}$ is [42] and the hyperfine coupling spin Hamiltonian for site $\mathbf{A}$ takes the form

$$
\hat{\mathcal{H}}_{A}^{\mathrm{HFC}}=A \mathbb{S}_{A}^{Z_{A}} I_{A}^{Z_{A}},
$$

with

$$
\frac{A}{g}=\frac{A_{J}}{g_{J}},
$$

where $g_{J}$ is the Landé $g$ factor $[=5 / 4$ for $\mathrm{Ho}(\mathrm{III})]$. The value of $A_{J}$ for Ho(III) was determined by EPR measurements in ethyl sulfates crystal to be $815 \mathrm{MHz}$ [42]. This value was confirmed by further experiments, in $\mathrm{LiHoF}_{4}$ by EPR [43] and optical spectroscopy [44] and in yttrium hydroxide by spin echo NMR [45]. Recently, $A_{J}$ was deduced from EPR in $\left[\mathrm{Ho}(\mathrm{III})\left(\mathrm{W}_{5} \mathrm{O}_{18}\right)_{2}\right]^{9-}$ and found to be slightly larger with $830 \mathrm{MHz}$ [37]. It shows that the HFC constant varies little with the environment, due to the ionic character of the bond. In the following, we will take $A_{J}=830 \mathrm{MHz}$ and with $g=15$, one gets $A=9960 \mathrm{MHz}$. The nuclear quadrupole and the nuclear Zeeman interactions are neglected since they are much smaller than the HFC term. To reduce the model space to the ground doublet, one neglects the effect of the excited states, which bring terms above the Ising-like magnetism. The use of the following spin Hamiltonian is restricted to the low temperature domain.

The two local HFC operators $\hat{\mathcal{H}}_{A / B}^{\mathrm{HFC}}$ are added to the electronic Hamiltonian

$$
\hat{\mathcal{H}}_{T}=\hat{\mathcal{H}}_{\mathrm{el}}+\hat{\mathcal{H}}_{A}^{\mathrm{HFC}}+\hat{\mathcal{H}}_{B}^{\mathrm{HFC}} .
$$

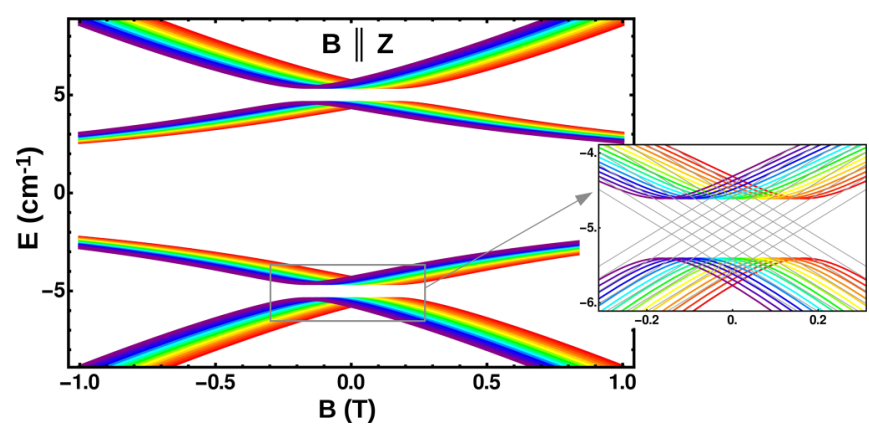

FIG. 9. Zeeman diagram for the dinuclear complex including nuclear degrees of freedom for a magnetic field in direction $Z$. Parameters are given in Fig. 8. The colors denote the value of $M_{I A}+M_{I B}$; from purple to red, $M_{I A}+M_{I B}$ varies from 7 to -7 . The inset zooms in the avoided crossing region. In gray, the case of the mononuclear complex with $\Delta=0$ is depicted. 


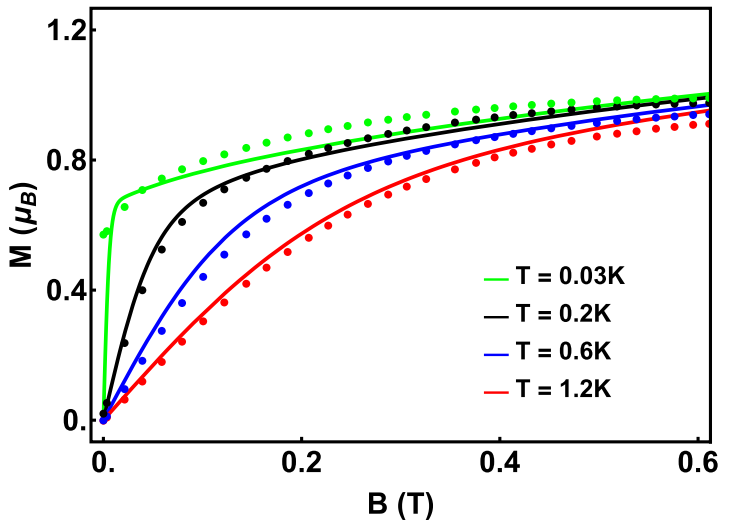

FIG. 10. Experimental magnetization along the easy axis of magnetization (points) at $\omega=0.002 \mathrm{~T} / \mathrm{s}$ and theoretical curves (lines) obtained with the Hamiltonian of Eq. (17) with the same parameters as Fig. 8 and a thermal distribution at different temperatures.

The basis set for the binuclear compound is spanned by the 256 $\left| \pm, M_{I}\right\rangle_{A} \otimes\left| \pm, M_{I}\right\rangle_{B}$ states. According to the Hamiltonian of Eq. (15), states with different values of $M_{I}$ do not couple. The Zeeman diagram for the binuclear compound including the nuclear degrees of freedom is shown in Fig. 9.

\section{MODELING OF THE HYSTERESIS}

In Fig. 4 the curves with a sweep of $0.002 \mathrm{~T} / \mathrm{s}$ do not show hysteresis except for the lowest temperature $T=0.03 \mathrm{~K}$. The thermal equilibrium is reached and the magnetization is deduced from the Hamiltonian (17) with a Boltzmann population; it is represented in Fig. 10 and is in good agreement with the experimental data. The nuclear sublevels are separated by an energy of $\mathcal{A} / 2=0.16 \mathrm{~cm}^{-1}$; not all of these sublevels are populated at $T=0.03 \mathrm{~K}$. The energies of the $M_{I}$ manifold as a function of magnetic field are not parallel such that the sublevels do not have the same electronic magnetization; the total magnetization will consequently depend on the relative population of the $M_{I}$ sublevels. As soon as a field is applied, the degeneracy of the $\pm M_{I}$ states is removed (see inset of Fig. 9) and the magnetization increases abruptly since only the ground sublevel is populated. On the contrary, at $T=1.2 \mathrm{~K}$ all the $M_{I}$ sublevels are populated and the increase of the magnetization arises from the smooth change of magnetization of the sublevels but not from their relative population. At $T=0.03 \mathrm{~K}$, the increase of magnetization is due to the thermal population of the nuclear sublevels while at $T=2 \mathrm{~K}$, all nuclear sublevels are equally populated and the excited electronic states $(| \pm \mp\rangle$ and $| \pm \pm\rangle)$ contribute to the temperature dependence of the magnetization.

The hysteresis arises because when varying the field, the system remains in the same state, even when becoming metastable, since there is no coupling between the states with different values of $M_{I}$. The largest hysteresis would be obtained by following the $M_{I}=7$ sublevel for decreasing field and the $M_{I}=-7$ sublevel for increasing field (see Fig. 7). The magnetization of the $M_{I}= \pm 7$ sublevels represents the envelope in which the hysteresis must lie. In Fig. 11 the magnetization of $M_{I}= \pm 7$ sublevels are shown for different values of $\Delta, M^{Z}$, and $J$ in the case of a symmetrical complex with $\Delta_{A}=\Delta_{B}=\Delta$. The effect of $\Delta$ is the progressive mixing between the electronic states and consequently a smoothing of the magnetization: the larger $\Delta$ is, the less abrupt the change in the magnetization and narrower the hysteresis is. An increase in $\Delta$ smoothens the loop but does not impact its width, an increase in $M^{Z}$ sharpens and shrinks the loop while a positive (negative) $J$ sharpens (smoothens) the loop without affecting its width. The effects of $\Delta$ and $J$ are very similar such that it is difficult to deconvolute the effects of $\Delta$ and $J$ from the experimental spectra.

As expected for a noncollective phenomenon, the largest hysteresis loop is obtained with the smallest temperature $T=$ $0.03 \mathrm{~K}$ and the largest sweep rate $0.28 \mathrm{~T} / \mathrm{s}$ and originates from the population of metastable states; this magnetic hysteresis is represented in Fig. 12 with the magnetization of the $M_{I}$ sublevels of the ground manifold of Fig. 9. As stated in the previous section, the Hamiltonian of Eq. (17) does not couple states with different values of $M_{I}=M_{I A}+M_{I B}$ and these sublevels may be metastable, up to some point. The comparison of the experimental hysteresis curve with this manifold of curves permits us to determine the pathway of the transition between $M_{I}=7$ and -7 sublevels. For $B>0$, the lowest state corresponds to $M_{I}=7$ (purple curve) and for $B<0$ to $M_{I}=-7$ (red curve). Since there is no coupling between the $M_{I}$ states, the system may stay in a metastable state. Starting from $B>0$ and decreasing the field, the experimental curve follows the purple curve until $B=-0.05 \mathrm{~T}$ corresponding to the maximum of energy of this $M_{I}$ state; then there is a deexcitation to the green curve $\left(M_{I A}+M_{I B}=0\right)$. Starting from $B<0$ and increasing the field, the experimental points follow the red line up to $B=0.05 \mathrm{~T}$ and there a deexcitation to lower levels occur, on
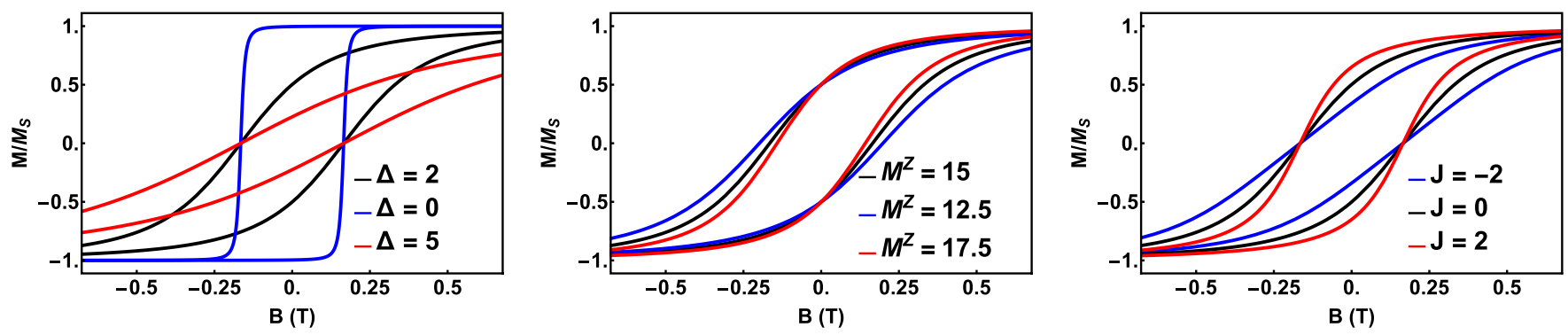

FIG. 11. Effect of $\Delta, M^{Z}$, and $J$ on the envelope of the magnetization curves. Left: $M^{Z}=15, J=0$; center: $\Delta=2 \mathrm{~cm}^{-1}, J=0$; right: $M^{Z}=15, \Delta=2 \mathrm{~cm}^{-1} . \Delta=\Delta_{A}=\Delta_{B}$. 


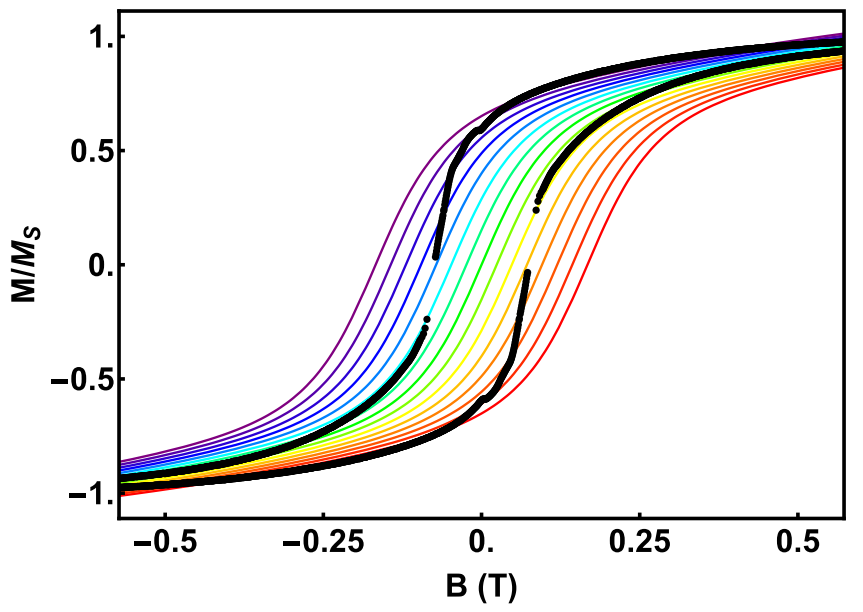

FIG. 12. Experimental relative magnetization along the easy axis of magnetization (black points) at $T=0.03 \mathrm{~K}$ and $\omega=0.28 \mathrm{~T} / \mathrm{s}$ and the magnetization of the lowest manifolds calculated with the Hamiltonian of Eq. (17) with the same parameters as Fig. 8. The colors denote the value of $M_{I A}+M_{I B}$; from purple to red, $M_{I A}+M_{I B}$ varies from 7 to -7 .

the green curve as well. The effect of the zero-field gap $\Delta_{B}$ is the progressive mixing between the electronic states $|--\rangle$ and $|+-\rangle$ and leads to a smoothing of the magnetization as compared to the previous studies: the larger the gap is, the less abrupt the change in the magnetization is and the narrower the hysteresis is. The purple and red curves in Fig. 12 denote the largest loop corresponding to the Zeeman diagram; the experimental curve lies within the envelope of the theoretical ones. In order for the hysteresis to occur, the HFC sublevels may be partially populated: that is why it disappears for temperatures larger than $0.5 \mathrm{~K}$.

\section{CONCLUSION}

In this article we have reported the magnetic hysteresis observed in a binuclear Ho(III) complex. Using a combined characterization of x-ray crystallography, magnetometry, and first-principles calculations, a spin Hamiltonian has been proposed for the dinuclear species, describing both electronic and nuclear magnetic degrees of freedom. According to the Hamiltonian parameters that match all the available experimental data, each Ho(III) ion is a non-Kramers doublet, the octacoordinated Ho(III) ion with a rather small gap of $0.8 \mathrm{~cm}^{-1}$ and the heptacoordinated Ho(III) ion with a larger gap of $10 \mathrm{~cm}^{-1}$, with a small ferromagnetic exchange coupling between the two sites. The driving force of the magnetic hysteresis is shown to be the metastability of the hyperfine levels while its shape is determined by the electronic parameters, energy gaps in the local doublets, and magnetic coupling.

\section{ACKNOWLEDGMENTS}

F.K. thanks the Scientific Council of the LCPQ for attributing his Ph.D. grant. This work is partially financed by ANR-project MolQuSpin 13- BS10-0001-03. This work is supported by a public grant overseen by the French National Research Agency (ANR) as part of the "Investissements d' Avenir" program "Labex NanoSaclay" (reference ANR10-LABX-0035). We acknowledge the Université Paris-Sud, UPS, RégionIledeFranceSESAMEprogram 2012no.12018501 and CNRS. T.M. thanks the IUF (Institut Universitaire de France) for financial support.
[1] D. N. Woodruff, R. E. P. Winpenny, and R. A. Layfield, Chem. Rev. 113, 5110 (2013).

[2] M. N. Leuenberger and D. Loss, Physica E (Amsterdam) 10, 452 (2001).

[3] M. N. Leuenberger and D. Loss, Nature (London) 410, 789 (2001).

[4] M. Urdampilleta, S. Klyatskaya, J. P. Cleuziou, M. Ruben, and W. Wernsdorfer, Nat. Mater. 10, 502 (2011).

[5] A. Candini, G. Lorusso, F. Troiani, A. Ghirri, S. Carretta, P. Santini, G. Amoretti, C. Muryn, F. Tuna, G. Timco, E. J. L. McInnes, R. E. P. Winpenny, W. Wernsdorfer, and M. Affronte, Phys. Rev. Lett. 104, 037203 (2010).

[6] G. Aromi, D. Aguila, P. Gamez, F. Luis, and O. Roubeau, Chem. Soc. Rev. 41, 537 (2012).

[7] J. Ferrando-Soria, E. M. Pineda, A. Chiesa, A. Fernandez, S. A. Magee, S. Carretta, P. Santini, I. J. Vitorica-Yrezabal, F. Tuna, G. A. Timco, E. J. L. McInnes, and R. E. P. Winpenny, Chem 1, 727 (2016).

[8] J. Ferrando-Soria, E. M. Pineda, A. Chiesa, A. Fernandez, S. A. Magee, S. Carretta, P. Santini, I. J. Vitorica-Yrezabal, F. Tuna, G. A. Timco, E. J. L. McInnes, and R. E. P. Winpenny, Nat. Commun. 7, 11377 (2016).

[9] C. Y. Chow, H. Bolvin, V. E. Campbell, J. W. Guillot, R. Kampf, W. Wernsdorfer, F. Gendron, J. Autschbach, V. Pecoraro, and T. Mallah, Chem. Sci. 6, 4148 (2015).
[10] N. Ishikawa, M. Sugita, and W. Wernsdorfer, J. Am. Chem. Soc. 127, 3650 (2003).

[11] Y. C. Chen, J. L. Liu, W. Wernsdorfer, D. Liu, L. F. Chibotaru, X. M. Chen, and M. L. Tong, Angew. Chem., Int. Ed. Engl. 56, 4996 (2017).

[12] F. Habib and M. Murugesu, Chem. Soc. Rev. 42, 3278 (2013).

[13] F. Pointillart, B. Le Guennic, O. Maury, S. Golhen, O. Cador, and L. Ouahab, Inorg. Chem. 52, 1398 (2013).

[14] H. Ke, S. Zhang, X. Li, Q. Wei, G. Xie, W. Wang, and S. Chen, Dalton Trans. 44, 21025 (2015).

[15] S. F. Xue, Y. N. Guo, L. Ungur, J. K. Tang, and L. F. Chibotaru, Chem. Eur. J. 21, 14099 (2015).

[16] Y.-L. Wang, C.-B. Han, Y.-Q. Zhang, Q.-Y. Liu, C.-M. Liu, and S.-G. Yin, Inorg. Chem. 55, 5578 (2016).

[17] L. Zhang, J. Jung, P. Zhang, M. Guo, L. Zhao, J. Tang, and B. Le Guennic, Chem. Eur. J. 22, 1392 (2016).

[18] L. Zhang, Y.-Q. Zhang, P. Zhang, L. Zhao, M. Guo, and J. Tang, Inorg. Chem. 56, 7882 (2017).

[19] J. Kan, H. Wang, W. Sun, W. Cao, J. Tao, and J. Jiang, Inorg. Chem. 52, 8505 (2013).

[20] W. Yu, F. Schramm, E. M. Pineda, Y. Lan, O. Fuhr, J. Chen, H. Isshiki, W. Wernsdorfer, M. Wulfhekel, and W. Ruben, Beilstein J. Nanotechnol. 7, 126 (2016).

[21] H. Tian, S. S. Bao, and L. M. Zheng, Chem. Commun. 52, 2314 (2016). 
[22] Q. Chen, F. Ma, Y. S. Meng, H. L. Sun, Y. Q. Zhang, and S. Gao, Inorg. Chem. 55, 12904 (2016).

[23] T. Lacelle, G. Brunet, A. Pialat, R. J. Holmberg, Y. La, B. Gabidulin, I. Korobkov, W. Wersdorfer, and M. Murugesu, Dalton Trans. 46, 2471 (2017).

[24] F. S. Guo and R. Layfield, Chem. Commun. 53, 3130 (2017).

[25] J. D. Leng, J. L. Liu, W. Q. Lin, S. Gomez-Coca, D. Aravena, E. Ruiz, and M. L. Tong, Chem. Commun. 49, 9341 (2013).

[26] See Supplemental Material at http://link.aps.org/supplemental/ 10.1103/PhysRevB.96.214427 for experimental and computational details and more SO-CASSCF results.

[27] W. Wernsdorfer, Supercond. Sci. Technol. 22, 064013 (2009).

[28] F. Aquilante, L. De Vico, N. Ferré, G. Ghigo, P.-A. Malmqvist, P. Neogrády, T. B. Pedersen, M. Pitonak, M. Reiher, B. Roos, M. Serrano-Andrés, M. Urban, V. Veryazov, and R. Lindh, J. Comput. Chem. 31, 224 (2010).

[29] B. O. Roos, R. Lindh, P. Malmqvist, V. Veryazov, and P. O. Widmark, J. Phys. Chem. A 108, 2851 (2004).

[30] B. O. Roos, R. Lindh, P. Malmqvist, V. Veryazov, and P. O. Widmark, Chem. Phys. Lett. 409, 295 (2005).

[31] B. O. Roos, P. R. Taylor, and P. E. M. Siegbahn, Chem. Phys. 48, 157 (1980).

[32] P.-A. Malmqvist, B. O. Roos, and B. Schimmelpfennig, Chem. Phys. Lett. 357, 230 (2002).
[33] B. A. Hess, C. M. Marian, U. Wahlgren, and O. Gropen, Chem. Phys. Lett. 251, 365 (1996).

[34] H. Bolvin, Chem. Phys. Chem. 7, 1575 (2006).

[35] L. Ungur and L. F. Chibotaru, Chem. Eur. J. 23, 3708 (2017).

[36] S. Vancoillie, F. Neese, L. Rulisek, and K. Pierloot, J. Phys. Chem. A 113, 6149 (2009).

[37] S. Ghosh, S. Datta, L. Friend, S. Cardona-Serra, A. Gaita-Ariño, E. Coronado, and S. Hill, Dalton Trans. 41, 13697 (2012).

[38] N. Ishikawa, M. Sugita, T. Okubo, N. Tanaka, T. Lino, and Y. Kaizu, Inorg. Chem. 42, 2440 (2003).

[39] J. S. Griffith, Phys. Rev. 132, 316 (1963).

[40] H. Bolvin and J. Autschbach, Handbook of Relativistic Quantum Chemistry (Springer, Berlin, 2017) Chapter on Relativistic methods for calculating Electron Paramagnetic Resonance (EPR) parameters.

[41] A. Abragam and B. Bleaney, Electronic Paramagnetic Resonance of Transition Ions (Clarendon Press, Oxford, 1970).

[42] J. M. Baker and B. Bleaney, Proc. R. Soc. London Sect. A 245, 156 (1958).

[43] J. Magariño, J. Tuchendler, P. Beauvillain, and I. Laursen, Phys. Rev. B 21, 18 (1980).

[44] N. I. Agladze and M. N. Popova, Solid State Commun. 55, 1097 (1985).

[45] D. S. P. Bunbury, C. Carboni, R. L. Cone, M. A. H. McCausland, S. Mroczkowski, and J. W. Ross, J. Phys.: Condens. Matter 7, 6125 (1995). 